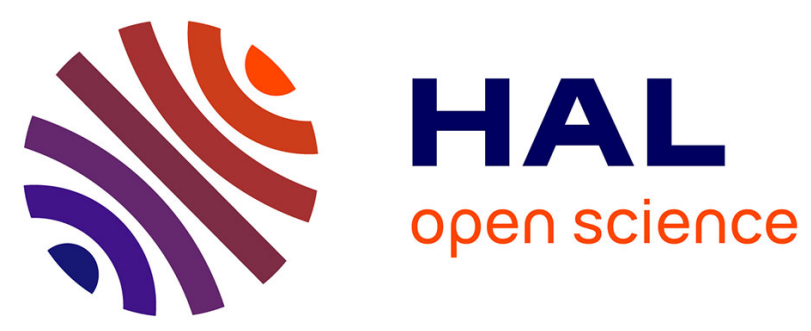

\title{
Using Heterogeneous Multilevel Swarms of UAVs and High-Level Data Fusion to Support Situation Management in Surveillance Scenarios
}

Pascal Bouvry, Serge Chaumette, Grégoire Danoy, Gilles Guerrini, Gilles Jurquet, Achim Kuwertz, Wilmuth Müller, Martin Rosalie, Jennifer Sander

\section{To cite this version:}

Pascal Bouvry, Serge Chaumette, Grégoire Danoy, Gilles Guerrini, Gilles Jurquet, et al.. Using Heterogeneous Multilevel Swarms of UAVs and High-Level Data Fusion to Support Situation Management in Surveillance Scenarios. International Conference on Multisensor Fusion and Integration for Intelligent Systems (MFI 2016), Sep 2016, Baden-Baden, Germany. 10.1109/MFI.2016.7849525 . hal-01360162

\section{HAL Id: hal-01360162 \\ https://hal.science/hal-01360162}

Submitted on 5 Sep 2016

HAL is a multi-disciplinary open access archive for the deposit and dissemination of scientific research documents, whether they are published or not. The documents may come from teaching and research institutions in France or abroad, or from public or private research centers.
L'archive ouverte pluridisciplinaire HAL, est destinée au dépôt et à la diffusion de documents scientifiques de niveau recherche, publiés ou non, émanant des établissements d'enseignement et de recherche français ou étrangers, des laboratoires publics ou privés. 


\title{
Using Heterogeneous Multilevel Swarms of UAVs and High-Level Data Fusion to Support Situation Management in Surveillance Scenarios
}

\author{
Pascal Bouvry ${ }^{1}$, Serge Chaumette ${ }^{2}$, Grégoire Danoy ${ }^{1}$, Gilles Guerrini ${ }^{3}$, Gilles Jurquet ${ }^{3}$, \\ Achim Kuwertz ${ }^{4}$, Wilmuth Müller ${ }^{4}$, Martin Rosalie ${ }^{1,2}$ and Jennifer Sander ${ }^{4}$
}

\begin{abstract}
The development and usage of Unmanned Aerial Vehicles (UAVs) quickly increased in the last decades, mainly for military purposes. This technology is also now of high interest in non-military contexts like logistics, environmental studies and different areas of civil protection. While the technology for operating a single UAV is rather mature, additional efforts are still necessary for using UAVs in fleets (or swarms). The Aid to SItuation Management based on MUltimodal, MUltiUAVs, MUltilevel acquisition Techniques (ASIMUT) project which is supported by the European Defence Agency (EDA) aims at investigating and demonstrating dedicated surveillance services based on fleets of UAVs. The aim is to enhance the situation awareness of an operator and to decrease his workload by providing support for the detection of threats based on multi-sensor multi-source data fusion. The operator is also supported by the combination of information delivered by the heterogeneous swarms of UAVs and by additional information extracted from intelligence databases. As a result, a distributed surveillance system increasing detection, high-level data fusion capabilities and UAV autonomy is proposed.
\end{abstract}

\section{INTRODUCTION}

The development and usage of Unmanned Aerial Vehicles (UAVs) quickly increased in the last decades, mainly for military purposes [1]. This technology is also now of high interest in non-military contexts like logistics, environmental studies and different areas of civil protection, for example rescue missions support, fire fighting, police duties [2]. While the technology for operating a single UAV is rather mature, additional efforts are still necessary for using UAVs in fleets (or swarms) [3], [4], [5].

ASIMUT (Aid to SItuation Management based on MUltimodal, MUltiUAVs, MUltilevel acquisition Techniques), a two-year project which started in March 2015, is supported by the EDA (European Defence Agency). The project is implemented by a consortium of five partners: Thales

\footnotetext{
*The authors acknowledge the support of the ASIMUT project A-1341RT-GP, which is coordinated by the European Defence Agency (EDA) and partially funded by eight contributing Members (Austria, France, Germany, Italy, Luxembourg, The Netherlands, Poland and Sweden) in the framework of the Joint Investment Programme on Innovative Concepts and Emerging Technologies 2.

${ }^{1}$ University of Luxembourg, SnT/FSTC-CSC, Luxembourg

pascal.bouvryeuni.lu gregoire.danoyduni.lu martin.rosalieduni.lu

${ }^{2}$ Univ. Bordeaux, LaBRI, UMR 5800, F-33400, Talence, France serge. chaumetted labri.fr

${ }^{3}$ Thales Airborne Systems, Pessac, France gilles.guerrini@fr.thalesgroup.com gilles.jurquetefr.thalesgroup.com

${ }^{4}$ Fraunhofer IOSB, Karlsruhe, Germany

achim.kuwertzeiosb. fraunhofer. de

wilmuth.muellerdiosb. fraunhofer.de

jennifer.sandereiosb. fraunhofer. de
}

Systèmes Aéroportés, Laboratoire Bordelais de Recherche en Informatique of the University of Bordeaux, Fraunhofer IOSB, the University of Luxembourg and the French SME Fly'n'Sense. It aims at researching and demonstrating dedicated surveillance services being essentially based on fleets of heterogeneous UAVs. The aim is to enhance the situation awareness of operators and to decrease their workload by providing a model supporting detection of threats based on multi-sensor multi-source data fusion.

The notion of heterogeneous UAVs means different types of UAVs as well as different types of sensors (electrooptical, infrared, etc.) carried by the UAVs. Collaboration between UAVs evolving at different altitudes, i.e., between large UAVs covering the considered area from a high altitude with coarse acquisition capabilities and low altitude multirotor UAVs for precise close to the field measurements, is also taken into account.

Because of the hostile environment in which the aircrafts will be deployed, the flight plans cannot be computed in an offline manner by a central entity and distributed prior to the start of the mission. Autonomous UAVs taking their own decisions concerning their movement are thus more suitable. This approach has to be addressed adequately taking selforganization processes, collective intelligence and wireless networking issues into account.

The ASIMUT system supports operators through the combination of information coming from an airborne surveillance system based on heterogeneous swarms of UAVs and additional information extracted from intelligence databases. By providing such an automated assistance to human operators (in terms of high-level data fusion), their capabilities for making efficient and effective decisions can be improved thanks to increased situation awareness and lower workload.

The ASIMUT project aims at a balanced combination of different measures, framed by the types of threats and by the operator's reaction capabilities. To reach this aim, different innovative algorithms and approaches addressing maneuverability and autonomy, reliance on extended onboard situational awareness, information sharing, and highlevel data fusion based on promising concepts from artificial intelligence and model-based reasoning, are combined profitably in the distributed surveillance system.

The remainder of this paper is organized as follows. In Sec. II, we give an overview on concepts and system design of ASIMUT: we sketch our basic approach and present the main findings with regard to the ASIMUT structure and the resulting ASIMUT system. The following sections give an 


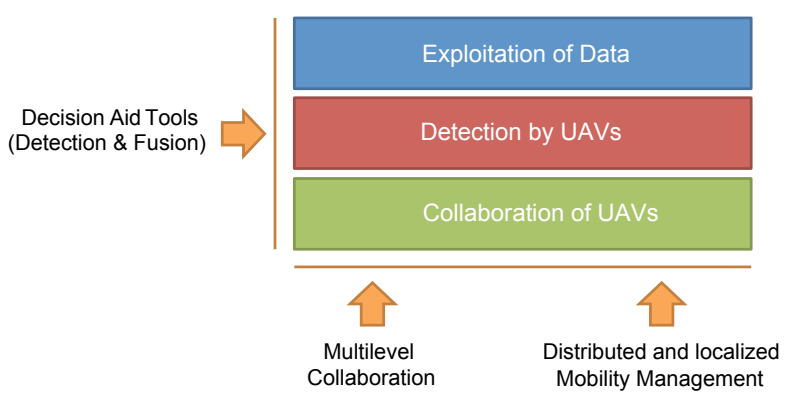

Fig. 1. ASIMUT structure.

overview on the main approaches and algorithms used within the ASIMUT system. Sec. III addresses FMV (Full Motion Video) analysis and focuses on moving target detection. Sec. IV presents the work done with regard to autonomy, mobility, and resilience. In Sec. V, our approaches for high-level data fusion are presented. Before we give our conclusions in Sec. VII, Sec. VI addresses our concepts and future plans with regard to system evaluation.

\section{CONCEPT AND System Design}

For working out concept and design of the ASIMUT system, the operational and technical needs for surveillance in the military domain have been identified and analyzed in detail. The basis has been the development of operationally relevant use cases, the definition of a basic layered model structure, and a formal functional analysis of the system as a whole. In what follows, the main findings with regard to the ASIMUT structure and the resulting ASIMUT system are presented.

\section{A. ASIMUT Structure}

Fig. 1 gives a coarse view on the ASIMUT layered structure. Different approaches are integrated in three layers, each layer serving specific purposes:

\section{- Collaboration of UAVs}

This layer enables the collaboration between the UAVs. This is done by UAV swarm flight plan optimization under consideration of the external environment.

\section{- Detection by UAVs}

This layer enables the UAVs to gather the information needed about multiple objects, events, and their relationships. To achieve this goal, each UAV carries a specific payload to perform the air-ground surveillance. The autonomy of the UAV swarm makes it possible to decide which UAVs are relevant for performing a specific analysis tasked.

\section{- Exploitation of Data}

This layer enables further exploitation of the data gathered by the UAVs and of additional information from intelligence databases and also to relate them together. It is then possible to generate data and information of higher quality and deduce additional information. Specific means like FMV analysis and higher level data fusion techniques are provided to achieve this goal.

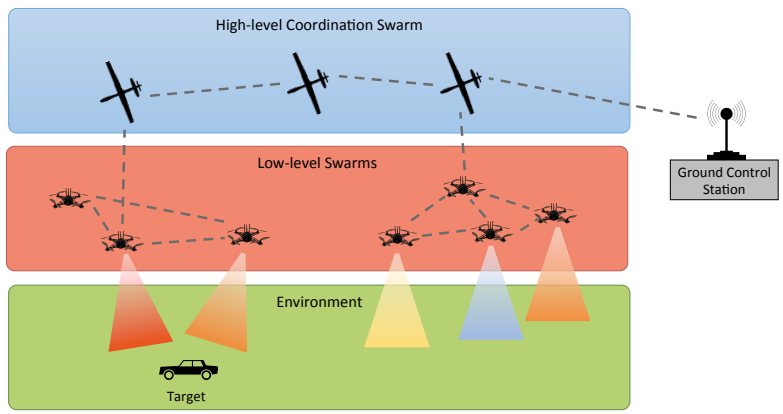

Fig. 2. Multilevel swarms of UAVs collaborating.

\section{B. ASIMUT System}

The ASIMUT system is a distributed surveillance system composed of a Command and Control (C2) system and of a set of heterogeneous UAVs that constitute swarms. The swarms can operate in two different modes: area surveillance and moving object (target) tracking. As illustrated Fig. 2, there is multilevel cooperation between the UAVs, a HighLevel Coordination Swarm (HLCS) commanding several Low-Level Swarms (LLSs). The LLSs have reduced onboard processing capabilities. The HLCS have more powerful processing capabilities, but still less than the $\mathrm{C} 2$ system. The C2 system is composed of a Ground Control Station (GCS) in charge of the control of the missions of the UAV swarms and of an Intelligence Centre (IC). The IC (for illustration purposes not depicted in Fig. 2) is responsible for correlating and aggregating the information generated by the UAVs with information available from other sources, mainly intelligence databases. The GCS controls the swarms by assigning them specific missions while the actual mission planning process is performed by the swarms themselves. The IC performs high-level data fusion for generating decision support that is presented to the commander. The intelligence personnel in the IC has access to internal and external intelligence databases, high-level data fusion tools, and to the tracks generated by the UAV swarms.

\section{Moving TARget Detection IN A VIDEO}

For their tactical missions, the UAVs are equipped with various on-board sensors including long-range radar and electro-optical/infrared cameras. Both of these payloads are associated with geolocation and allow effective surveillance and intelligence. Our objective is to monitor the movements of ground vehicles like trucks, cars, motorbikes and to recognize the targets of interest (according to the tasks to be fulfilled). This is done based on algorithms allowing the automatic detection of moving ground targets from a realtime video acquisition performing this detection for small targets (i.e., when the moving target in a video has a size that is lower than two pixels). The data flow is dynamic, the camera moves, and we need to make a distinction between the ground and objects whose speed and direction evolve separately. 


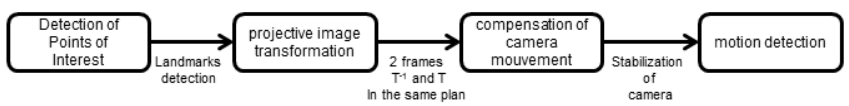

Fig. 3. Overview of the different steps within the process of automated detection of targets based on motion detection in full motion video.

To perform automatic detection of targets in a full motion video, the process has been divided into several steps (see Fig. 3): work on optical flow, detection of points of interest, projective image transformation, compensation for camera movement, motion detection.

The optical flow allows us to determine the motion vector of a pixel between two images of the same scene, i.e., between two consecutive frames in a video flow. This technique is widely used in the field of video processing for tracking an object by determining the transition matrix between the two images. Knowing the displacement of pixels, the interest is to have landmarks in the images. With them, an analysis can be done to determine the movement of objects relative to others. A first application is the segmentation of the video into different regions according to the motion vectors. In the ASIMUT project, algorithms are developed on basis of approaches from Lucas/Kanade [6] and Bouguet [7] to perform the detection and the tracking of moving targets in a recorded video or directly from a real-time video stream.

A point of interest is defined as a specific area of the image that has a property predefined by an image processing operator, for example corners. These operators are varied; the best known is the method of Harris [8] for corners, corresponding to an important change in the direction of an edge in an image. As for the optical flow, we are interested in landmarks between the two images. It is possible to correlate the points of interest to obtain the displacement vector. In addition, the method of Harris allows to obtain particularly stable and favorable points for optical flow computation to perform segmentation of regions based on their motion vector and to identify those which evolve independently.

According to the use cases, different picture transformations are possible and have to be handled. The projective transformation addresses translation, rotation and scaling. This transformation allows to transform an image into the coordinate space of another, stabilizing the video acquisition. In the context of a dynamic video, the acquisition conditions constantly change. With this operator, it can be compensated for and then it is possible to have a $T-1$ frame in the space of the frame $T$ and to be able to apply operators of motion detection as for a fixed camera. In order to find this transformation, we must know at least four points that are present in both images with the associated displacement vector. One can then define the same plane and find the corresponding transformation matrix. To avoid alteration of the transition matrix by outliers, the method of RANSAC [9] is used to eliminate these values.

For compensating the camera movement, we need to know the projective transformation between each frame of a video stream. The major part of the image is the background

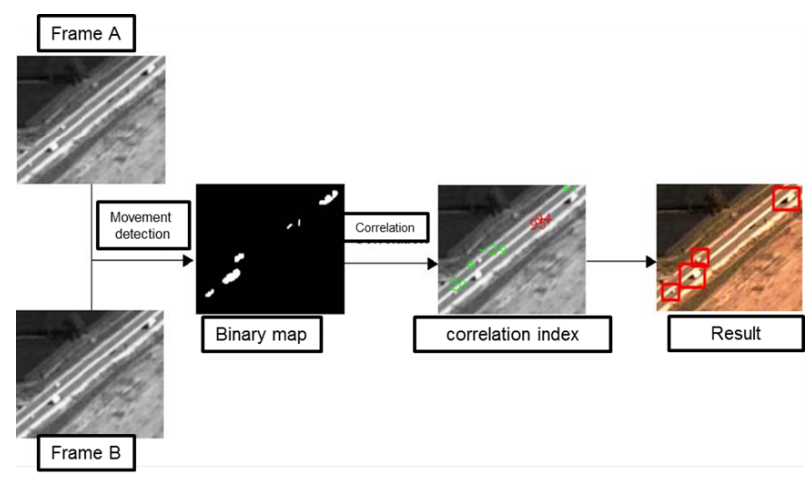

Fig. 4. Patterns of motion detection between two frames with the verification and the final result.

and does not correspond to moving objects. Based on this reasoning, a grid of points is used on the previous image whereas the major parts of them will correspond to the ground. Then, the optical flow is calculated by applying the method of Lucas/Kanade. The output is a set of vectors that allow to find the corresponding transformation. This is done by the least squares method coupled with RANSAC to eliminate false values. The space between each point and the margin can be configured, the advantage of this is to be able to control the number of points and therefore the computation time and the quality of the result. A second solution is to calculate the points of interest obtained by the Harris corner detector which will then be the points given for optical flow calculation. With the resulting motion vectors, the transformation can be found.

For motion detection, we now regard the position of the camera to be fixed, due to compensating for the camera movement. The absolute difference of the two images is considered with the aim to determine the color distance between each pixel. In practice, the noise of the optronic sensor alters this result. Therefore, we determine a threshold below which differences are interpreted as noise. This results in a binary image according to this condition. To eliminate noise, a morphological operator, Erosion, is applied as a binary mask for each pixel.

Fig. 4 presents an example of the global algorithm to perform automatic detection of targets.

\section{Autonomy, Mobility, Resilience}

In the ASIMUT project, the system is fully decentralized. The GCS only sends mission requirements to the HLCS. The latter has the capability to decide who will achieve this mission: the HLCS itself or the HLCS in collaboration with one LLS. The UAVs of the considered swarms will then autonomously decide the actions they have to plan to complete this mission. If the HLCS receives too many missions, it has to stop or wait until the completion of one or more missions before assigning new ones. As a consequence, the swarms control the sharing of the load. The swarms are chosen depending on their capabilities, mainly based on the sensors they embed and on their relative position to the objective. 

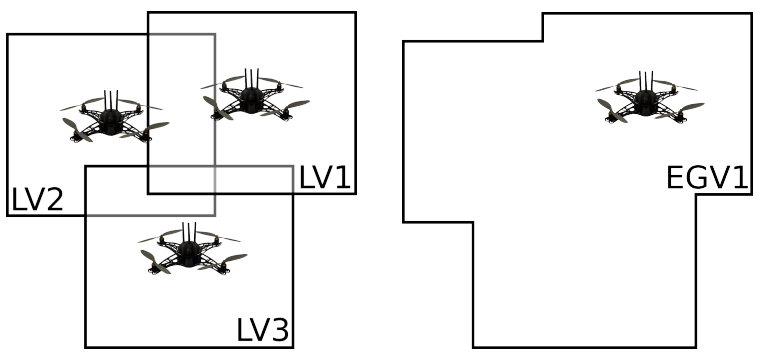

Fig. 5. Estimated Global View (EGV1) including the data collected from Local Views of other UAVs (LV2 and LV3) to improve decision making.

\section{A. Autonomous Retasking}

The LLSs need to support global retasking, i.e., retasking that involves all the multilevel swarms of the system. This need for retasking is not only because of their own behavior and internal events (e.g., loss of a UAV, refueling) but also because of the situation management requirements. In the latter case, this is the result of a HLCS decision. It might be necessary to retarget the mission and/or the role/location of each UAV depending on the sensors it embeds and on some situation management decision. The decision process of a UAV uses the data collected from the other UAVs of the swarm. This is done by building an estimated global view (Fig. 5) during the life cycle of a UAV; the latter being carried out perpetually. At any time, each UAVs receives the messages coming from other UAVs in its neighbourhood. Once per life cycle, it analyzes these messages to decide on the next action it has to perform autonomously and builds its own estimated global view.

\section{B. Mobility Model}

The UAVs mobility model is based on the ACO (Ant Colony Optimization) algorithm introduced by Dorigo [10]. The underlying idea is that ants use the environment to exchange information via pheromone deposit. The pheromone concentration then influences the behavior of UAVs (Fig. 6). This process is called stigmergy. UAVs deposit virtual repulsive pheromones in visited geographical areas so as to prevent other UAVs from revisiting the same places too often [11]. The drawback of the method is the usage of a random process when there is no pheromone in an area: randomness prevents the GCS operators from predicting the trajectories. To overcome this issue, we use a dynamical system with chaotic behavior, i.e. a deterministic process where the solution converges to a chaotic attractor. Here we propose to transpose this technique from the robotic to the UAV domain to address the path planning problem. Recently, the synchronization of chaotic dynamical systems have been used to obtain a better method for the coverage of an area [12]. Another approach relied on a discrete dynamical map (logistic map) in chaotic regime to generate series of bits [13]. These bits are then converted to positions points which permits to create a deterministic path planning for the robots.

In ASIMUT, another method is used to enforce a dynamical system in chaotic regime in our UAVs. More precisely, $\odot \odot \odot \odot \odot \odot \odot \odot \odot \odot \odot \odot \odot \odot \odot \odot \odot \odot \odot \odot$

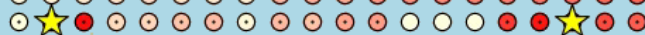
$\odot \odot \odot \odot \odot \odot \odot \odot \odot \odot ० ० ० 00 \odot \odot \odot \odot \odot$ $\odot \odot \odot \odot \odot \odot \odot$ $\odot \odot \odot$ ○ $\odot 00 \odot \odot \odot 00$

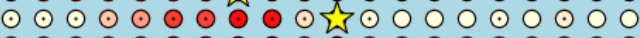

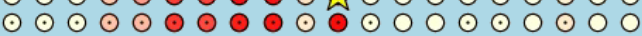
$\odot \odot \odot \odot \odot \odot \odot \odot \odot \odot \odot \odot \odot \odot \odot \odot \odot \odot ~ \odot ~ ○$ $\odot \odot \odot \odot \odot \odot \odot \odot \odot \odot \odot \odot \odot \odot \odot \odot \odot \odot \odot \odot$ $\odot \odot \odot \odot \odot \odot \odot \odot \odot \odot \odot \odot \odot \odot \odot \odot \odot \odot \odot \odot$

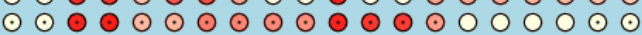
$\odot \odot \odot \odot \odot \odot \odot \odot \odot \odot \odot \odot \odot \odot \odot \odot \odot \odot \odot \odot$ $\odot \odot \odot \odot \odot \bar{น} \odot \odot \odot \odot \odot \odot \odot \odot \odot \odot \odot \odot \odot \odot$ $\odot \odot \odot \odot \odot \odot \odot \odot \odot \odot \odot \odot \odot \odot \odot \odot \odot \odot \odot \odot$ $\odot \odot \odot \odot \odot \odot \odot \odot \odot \odot \odot \odot \odot \odot \odot \odot \odot \odot \odot \odot$ $\odot \odot \odot \odot \odot \odot \odot \odot \odot \odot \odot \odot \odot \odot \odot \odot \odot \odot \odot \odot$ $\odot \odot \odot \odot \odot \odot \odot \odot \odot \odot \odot \odot \odot \odot \odot \odot \odot \odot \odot \overline{h ~}$ $\odot \odot \odot \odot \bar{W} \odot \odot \odot \odot \odot \odot \odot \odot \odot 乡 \odot \odot \odot \odot \odot$ $\odot \odot \odot \odot \odot \odot \odot \odot \odot \odot \odot \odot \odot \odot \odot \odot \odot \odot \odot \odot$

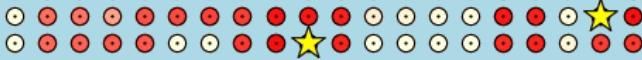

Fig. 6. LLS deposit of pheromone where stars represents UAVs and the light to dark shaded cells represents the low to high level of pheromones.

it consists of a Rössler system in a chaotic regime where the symbolic dynamic contains three symbols. Each one corresponds to a direction (left, ahead, and right) that a UAV can take when there is no pheromone to guide it. This ensures a deterministic choice that can be monitored directly by the GCS by just by having the same hardware and software as the UAVs. The main advantage of chaotic regime is that the process is deterministic while it remains unpredictable on a long term behavior. In our case, the use of the same devices guarantees to obtain the same digits. This helps the GCS operator to predict the behavior of UAVs when there is no pheromone to guide them.

\section{Resilience}

The main advantage of the decentralized model is that it handles the loss of UAVs. Each UAV decides on the actions to perform regularly which means that it analyzes its local environment by evaluating messages received from its neighbors. When the swarm looses a UAV, the others ones will no longer receive its messages and will accordingly decide on the appropriate actions. On the other hand, if a new UAV is tasked by the GCS to join this swarm its integration will be effective as soon as it has the capability to communicate its position and sensor data to the swarm members. This decentralized approach enables resilience and flexibility while UAVs are lost or added to reinforce the LLS actions.

\section{High-LeVel Data Fusion}

Sensor data collected from the LLSs and HLCS is processed and forwarded to the IC. Here, the results have to be combined with information from internal and external intelligence databases using methods from high-level data fusion (HLDF), with the aim of supporting the commander's situational awareness. HLDF denotes the process of integrating data and information on real-world entities observed in a 


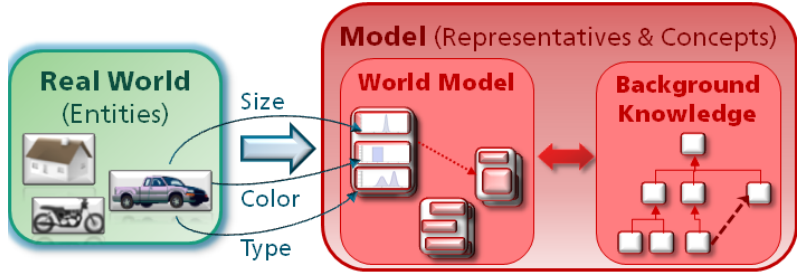

Fig. 7. Overview of the Object-Oriented World Model (OOWM), a framework for information representation, integration, and exchange under consideration of uncertainties.

dynamic environment into a consistent representation while, at the same time, studying the relationships between these entities [14]. According to the well-known JDL data fusion model [15], the assessment and integration of object-related information like their position and attributes (level 1) forms the basis for situation assessment (level 2), where the current relationships between objects are inferred, which then allows impact assessment (level 3).

In order to integrate heterogeneous data and information provided by different sensor systems, human observers and databases, as well as to account for different data formats and levels of abstraction, a unifying approach is needed in HLDF to ensure interoperability. Thus, as a first step and prerequisite for HLDF, available data and information has to be represented in an interoperable way, which in turn enables a consistent integration as a second step. Both steps can be tackled by applying methods from world modeling, an approach aiming at consistently representing and continuously updating available information about a given environment.

The Object-Oriented World Model (OOWM) [17] is a system designed for modeling a given environment in an object-oriented manner. Fig. 7 depicts an overview of the OOWM. It aims at representing the current state of an observed environment based on the contained objects. Each object is described by a set of attributes, which are the results of sensor measurements on certain object features. To account for uncertainties, attributes can be represented probabilistically. In addition, background knowledge on domain objects of interest can be provided, e.g., by domain experts. It is represented as an a priori domain model, e.g., an ontology. If new information becomes available, provided for example by sensors, methods from probabilistic information processing are applied for updating the object representations. Uncertainties resulting from measuring processes can thus be accounted for. In total, the OOWM acts as information hub, integrating information provided by different sources, and the contained information can be used as a basis for displaying a common operational picture (COP) of the observed environment.

For supporting HLDF in the ASIMUT project based on the OOWM approach, information from intelligence databases, such as formal reports, and data delivered by UAV swarms have to be integrated into the OOWM. The object-based representation of real-world entities can then be used to associate different data and information with the same real- world object. For example, on one hand, information about a specific truck observed near a suspicious building could be given in an intelligence report from a database. On the other hand, current real-time sensor data about the same truck can be provided by a UAV swarm, including track data. The UAV sensor data can then be associated with the report information by the OOWM and integrated into a single object representation. This covers the data fusion on JDL level 1. For JDL level 2, relationships between represented objects have to inferred. For this purpose, background knowledge has to be employed. The OOWM allows to store domain knowledge and general facts using descriptions logics and means of formal ontology, including rules representing implications. This background knowledge can be used to interrelate observed objects and to reason about them. For example, another report in the intelligence database could state that the previously mentioned building is being used as a site for manufacturing IEDs. In addition, another report could contain information about a current raid on a fertilizer factory. Combining these facts using rules modeled in the OOWM background knowledge, a reasoning tool can conclude that the truck currently observed by the UAV swarms might be loaded with explosives and therefore is intended to be used as an IED. The HLDF component in this case would inform operators in the IC about the potential threat and present them with its findings and lines of reasoning, for them to confirm or dismiss the fusion results.

The reasoning processes in HLDF can either be deterministic, e.g., using tools for ontology reasoning in a description logic like OWL DL, or make use of probabilistic extensions to description logic like the PR-OWL [18] language based on Bayesian networks. In addition, Markov logic networks [19] can be used to achieve probabilistic reasoning on top of a rule-based domain model. In domains where no sufficient amount of training data is available, the application of data-driven machine learning techniques for probabilistic reasoning is difficult. This is also true for the domain of military surveillance. Therefore, the model-based approach to reasoning described previously is taken in ASIMUT. Currently, a proof-of-concept implementation of the reasoning processes is being realized, further results for HLDF are expected at later project stages.

\section{Future Work: EVAluation}

Evaluation is essential to support the visibility of the developments, to materialize their benefits and thus validate their relevance. In ASIMUT, evaluation will be based on the developed use case scenarios describing the missions, their relative constraints and the types of aircrafts used along with respective aeronautic properties. The scenarios will permit to use all the capabilities of the system to achieve various missions including: area surveillance, target tracking and data collection. We also work on a proof-of-concept demonstrator.

Quality metrics will be defined to assess the performance of the ASIMUT system, with respect to: coverage of the surveyed area, connectivity of the communication graph, randomness of the surveillance movements and energy con- 


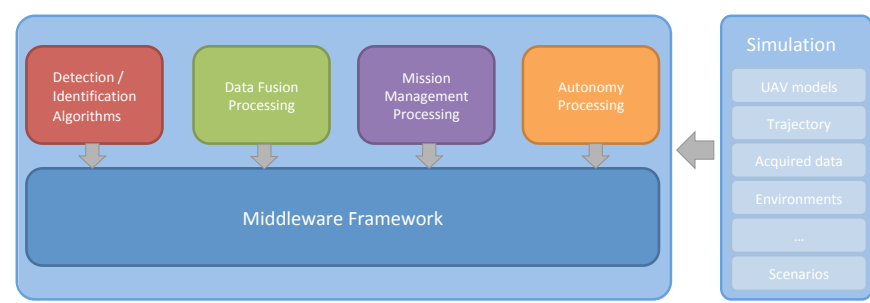

Fig. 8. ASIMUT evaluation architecture.

servation. The coverage of the surveyed area, i.e. what are the characteristics of a good or efficient coverage scheme and how to quantify them. Most promising benefit targets are the maximization of the average coverage frequency, the minimization of the area that is never covered, and even the minimization of the standard deviation of the coverage frequency. The connectivity of the communication graph, i.e. how well the UAV network should remain connected through time is also an important characteristics. The intrinsic dynamicity of the system requires the adaptation of static properties and measures, such as the number of connected components or the k-vertex connectivity for more resilient structures. The randomness of the surveillance movement will also be quantified, as the mobility of the UAVs should not be predictable. The energy conservation is also an important concern as it directly influences the autonomy of the surveillance system; the energy consumption of the system will thus be modeled and measured.

Furthermore, it is necessary to assess the improvement of decision quality and workload decrease for operators, which results from the automated assistance in high-level data fusion. Important characteristics for this assessment could for example be threat detection and false alarm rates as well as reaction times to such threats.

All the algorithms developed in ASIMUT will be combined in a common evaluation framework. Relying on stateof-the-art simulators, it will permit to evaluate the global chain from detection to data fusion, illustrated Fig. 8. Evaluation results shall be reported in future publications.

\section{CONCLUSION}

The ASIMUT project proposes, develops and validates beyond state-of-the-art technological building blocks for the enhancement of autonomy of surveillance missions based on multilevel UAV swarms. We propose new algorithmic approaches to address the different components of the functional chain of the underlying distributed system. The swarm decisional autonomy and mobility management take profit of past expertise on swarms of UAVs and new technologies for the application to multilevel swarms. Automatic processing of the detection of (even small) moving vehicles from realtime video acquisition by an electro-optical payload looks promising to decrease the operator workload. Providing automated assistance for human operators with regard to high-level data fusion tasks, ASIMUT significantly improves the capabilities of new algorithms for making efficient and effective decisions. Solutions for smart mission management and operator support have been developed. Information coming from the swarms can now be used to display the COP and other results within the ground control station and to compare the current situation with the planned one.

The most promising approaches implemented in the different algorithms that we have designed will be combined in a single demonstration framework and evaluated via stateof-the-art simulations. The objective will be to evaluate the benefits and relevance of the developments made; a preliminary step before a future system demonstration in a real environment representative of an operational environment.

\section{REFERENCES}

[1] K. P. Valavanis and G. J. Vachtsevanos, Handbook of Unmanned Aerial Vehicles. Springer, 2015

[2] I. Tchouchenkov, F. Segor, M. Kollmann, R. Schönbein, and T. Bierhoff, Detection, recognition and counter measures against unwanted UAVS, in Proc. 10th Future Security 2015, Security Research Conference, Fraunhofer Verlag, 2015, pp. 333-340.

[3] A. Bürkle, F. Segor, and M. Kollmann, Towards Autonomous Micro UAV Swarms, Journal of Intelligent \& Robotic Systems vol. 61, no. 1, pp. 339-353, 2011

[4] S. Chaumette, R. Laplace, C. Mazel, R. Mirault, A. Dunand, Y. Lecoutre, and J.-N. Perbet, CARUS, an operational retasking application for a swarm of autonomous UAVs: First re-turn on experience, in Military Communications Conference, MILCOM 2011, IEEE, 2011 pp. 2003-2010.

[5] O. K. Sahingoz, Mobile networking with UAVs: Opportunities and challenges, in Proc. 2013 Int. Conf. on Unmanned Aircraft Systems (ICUAS), 2013, pp. 933-941.

[6] B. D. Lucas and T. Kanade, An iterative image registration technique with an application to stereo vision, in Proc. of the 7th Int. Joint Conf. on Artificial intelligence, 1981, pp. 674-679.

[7] J.-Y. Bouguet. Pyramidal Implementation of the Lucas Kanade Feature Tracker, Description of the Algorithm, Intel Corporation, Microprocessor Research Labs, 2000.

[8] C. Harris and M. Stephens, A combined corner and edge detector, in Proc. of the 4th Alvey Vision Conference, 1988, pp. 147-151.

[9] M. A. Fischler and R. C. Bolles, Random sample consensus: A paradigm for model fitting with applications to image analysis and automated cartography, Comm. of the ACM, pp. 381-395, June 1981.

[10] M. Dorigo, Optimization, Learning and Natural Algorithms (in Italian), Ph.D. thesis, Dipartimento di Elettronica, Politecnico di Milano, Milan, Italy, 1992.

[11] E. Kuiper and S. Nadjm-Tehrani, Mobility Models for UAV Group Reconnaissance Applications, in Proc. of the 2006 Int. Conf. on Wireless and Mobile Communications, Bucharest, 2006, pp. 33-39.

[12] K. Fallahi and H. Leung, A cooperative mobile robot task assignment and coverage planning based on chaos synchronization, Int. Journal of Bifurcation and Chaos, vol. 20, no. 1, pp. 161-176, 2010.

[13] Ch. K. Volos, I. M. Kyprianidis, and I. N. Stouboulos, A chaotic path planning generator for autonomous mobile robots, Robotics and Autonomous Systems, vol. 60, no. 4, pp. 651-656, 2012.

[14] S. Das, High-Level Data Fusion. Boston: Artech House, 2008.

[15] A. N. Steinberg, C. L. Bowman, and F. E White, Jr., Revisions to the JDL data fusions models, Proc. of the 3rd NATO/IRIS Conference, Quebec City, Canada, 1998.

[16] J. Sander, A. Kuwertz, G. Schneider, and B. Essendorfer, ISR Analytics: Architectural and Methodic Concepts, in Proc. of the 2012 Workshop on Sensor Data Fusion: Trends, Solutions, Applications (SDF), Bonn, 2012, pp. 99-104.

[17] A. Kuwertz, J. Beyerer, Extending Adaptive World Modeling by Identifying and Handling Insufficient Knowledge Models, J. Appl. Log. (2016), http://dx.doi.org/10.1016/j.jal.2016.05.005 (in print).

[18] P. C. G. Costa, and K. B. Laskey, PR-OWL: A Framework for Probabilistic Ontologies, Frontiers in Artificial Intelligence and Applications, vol. 150, pp. 237-249, 2006

[19] P. Domingos, D. Lowd, S. Kok, H. Poon, M. Richardson, and P. Singla, Just Add Weights: Markov Logic for the Semantic Web. Uncertainty Reasoning for the Semantic Web I, Springer, 2015, pp. 1-25. 